\title{
Malnutrition and obesity in community-dwelling older adults: concomitant conditions? (PEN-3S study)
}

\author{
Teresa Madeira $^{1,2}$, Catarina Peixoto-Plácido ${ }^{1,2}$, Nuno Sousa-Santos ${ }^{3,2}$, Nuno Mendonça ${ }^{4}$, \\ Osvaldo Santos $^{1,2}$, Violeta Alarcão ${ }^{2,5}$, Paulo Jorge Nicola ${ }^{1,2}$, Carla Lopes ${ }^{6,7}$ and \\ João Gorjão Clara ${ }^{1,2}$ \\ ${ }^{1}$ Instituto de Medicina Preventiva e Saúde Pública, Faculdade de Medicina, Universidade de Lisboa, Lisbon, Portugal, \\ ${ }^{2}$ Instituto de Saúde Ambiental, Faculdade de Medicina, Universidade de Lisboa, Lisbon, Portugal, \\ ${ }^{3}$ Escola Superior de Saúde de Leiria, Instituto Politécnico de Leiria, Leiria, Portugal, \\ ${ }^{4}$ Institute for Ageing, Newcastle University, Newcastle upon Tyne, United Kingdom, \\ ${ }^{5}$ Centro de Investigação e Estudos de Sociologia (CIES-IUL), Instituto Universitário de Lisboa (ISCTE-IUL), \\ Lisbon, Portugal, \\ ${ }^{6}$ EPIUnit - Instituto de Saúde Pública da Universidade do Porto, Porto, Portugal and \\ ${ }^{7}$ Departamento de Ciências da Saúde Pública e Forenses e Educação Médica, Unidade de Epidemiologia da Faculdade \\ de Medicina da Universidade do Porto, Porto, Portugal
}

\begin{abstract}
Both malnutrition (which here refers to undernutrition) and obesity are prevalent in older adults, but they are frequently seen as mutually exclusive. In fact, a low body mass index (BMI) is one of the aetiological diagnostic criteria for malnutrition. On the contrary, the concomitant presence of malnutrition and obesity has been less studied. The aim of this study was to characterise the nutritional status of community-dwelling older adults ( $\geq 65$ years old) living in Portugal. The study included a nationally representative sample of randomly selected community-dwelling older adults. Trained nutritionists collected data through face-to-face structured interviews regarding sociodemographic characteristics, nutritional status and anthropometric measures (namely, weight and height), among other variables of the PEN-3S study. Nutritional status was assessed by the 18-item Mini Nutritional Assessment (full MNA ${ }^{\circledR}$ ), in which a lower score indicates worse nutritional status. The World Health Organisation's (WHO) BMI cut-offs for adults were followed (obesity: BMI $\geq 30 \mathrm{Kg} / \mathrm{m}^{2}$ ). MNA and BMI categories estimates $(95 \% \mathrm{CI}$ ) were obtained using Complex Samples analysis (SPSS $\left.^{\circledR} 24.0\right)$. Non-difference between sexes was analysed with Chi-square tests. Complete information was available for 1110 community-dwelling participants (mean age: $75.9 \pm 8.1$ years; $48.9 \%$ women; $71.4 \%$ attended school for $<5$ years). According to the MNA, $0.5 \%(95 \% \mathrm{CI}: 0.2-1.7)$ were classified as malnourished and $16.0 \%(12.9-19.7)$ were at risk of malnutrition. Following WHO's BMI criteria, $0.6 \%(0.2-1.5)$ had a BMI $\leq 18.5 \mathrm{Kg} / \mathrm{m}^{2}, 41.9 \%(37.9-46.0)$ had a BMI between $25-30 \mathrm{Kg} / \mathrm{m}^{2}$, and $36.7 \%$ (32.8-40.9) presented a $\mathrm{BMI} \geq 30 \mathrm{Kg} / \mathrm{m}^{2}$. The prevalence of risk of malnutrition was significantly higher for women $(20.1 \%, 95 \%$ CI: $15.4-25.9)$ than men $(10.4 \%, 7.6-14.1 ; p<0.001)$. The prevalence of obesity was also significantly higher for women $(42.2 \%$, $35.9-48.7$ versus $29.3 \%$, 24.8-34.2; $p=0.007)$. Moreover, $13.9 \%(9.2-20.4)$ were simultaneously at risk of malnutrition and had a $\mathrm{BMI} \geq 30 \mathrm{Kg} / \mathrm{m}^{2}$, while no one in this BMI category was classified as malnourished. Although appropriate BMI cut-offs for older adults are still uncertain, these results highlight that a high BMI does not exclude the risk of malnutrition, particularly in women. Therefore, health professionals should routinely screen for malnutrition using multi-component, validated screening tools, irrespective of the BMI. In fact, malnutrition is preventable if detected on time and effective interventions exist. The concomitant presence of malnutrition and obesity may pose additional challenges to the treatment.
\end{abstract}

\section{Conflict of Interest}

There is no conflict of interest. 